\title{
Transatlantica
}

Revue d'études américaines. American Studies Journal

2 | 2017

(Hi)stories of American Women: Writings and Rewritings / Call and Answer: Dialoguing the American West in France

\section{Carine Lounissi, Thomas Paine and the French Revolution}

Nathalie Caron

\section{OpenEdition}

\section{Journals}

Édition électronique

URL : https://journals.openedition.org/transatlantica/10512

DOI : 10.4000/transatlantica. 10512

ISSN : $1765-2766$

Éditeur

Association française d'Etudes Américaines (AFEA)

Référence électronique

Nathalie Caron, «Carine Lounissi, Thomas Paine and the French Revolution», Transatlantica [En ligne], 2 | 2017, mis en ligne le 19 avril 2019, consulté le 02 février 2023. URL : http://journals.openedition.org/ transatlantica/10512 ; DOI : https://doi.org/10.4000/transatlantica.10512

Ce document a été généré automatiquement le 2 février 2023.

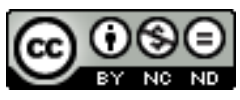

Creative Commons - Attribution - Pas d'Utilisation Commerciale - Pas de Modification 4.0 International - CC BY-NC-ND 4.0

https://creativecommons.org/licenses/by-nc-nd/4.0/ 


\title{
Carine Lounissi, Thomas Paine and the French Revolution
}

\author{
Nathalie Caron
}

\section{RÉFÉRENCE}

Carine Lounissi, Thomas Paine and the French Revolution. New York : Palgrave Macmillan, 2018. 321 pages, \$99.99, ISBN 978-3-319-75288-4

1 Carine Lounissi confirme dans ce nouvel ouvrage sa solide connaissance du corpus painien, qu'il s'agisse des textes de Paine ou des textes sur Paine. Elle accomplit, de plus, un travail précieux et trop rare: dans ce livre qu'elle a choisi de rédiger en anglais, elle combine, à partir de sa recherche sur une période de la vie de Paine encore largement lacunaire, les travaux des chercheurs britanniques et américains (et donc publiés en anglais) avec les travaux des chercheurs francophones qui sont, pour la totalité, non traduits. Sont ainsi commentés les travaux d'historiens de la France dont certains ont travaillé plus spécifiquement sur Paine (Yannick Bosc, Marc Belissa, en particulier, mais aussi Marcel Dorigny et Sophie Wahnich) ; d'historiens de la GrandeBretagne (tout particulièrement Gregory Clays, auteur de Thomas Paine: Social and Political Thought paru en 1991, dont un commentaire élogieux figure sur la quatrième de couverture) ; d'historiens des États-Unis (dont Bernard Vincent et Seth Cotlar). Carine Lounissi nous offre donc un large panorama de la recherche en anglais et en français sur Thomas Paine. Qui plus est, comme le souligne son éditeur, Thomas Paine and the French Revolution est le premier ouvrage en anglais consacré à la décennie française de Paine.

2 Le livre analyse la vie politique de Thomas Paine au cours des dix années qu'il passa à Paris, entre 1791 et 1802, année de son départ pour les États-Unis. Carine Lounissi évacue l'idée assez communément partagée selon laquelle Paine, qui ne parlait pas le français, ne prenait pas toute la mesure des enjeux de l'époque. Consciente des multiples appropriations dont Paine a fait l'objet, par ses contemporains comme par les 
historiens, elle cherche plutôt à évaluer le rôle que l'auteur de Rights of Man joua au cours de la période révolutionnaire française, autrement dit de la première République française, de sa mise en place en 1792 jusqu'au Consulat (entre 1799 et 1802), en passant par les quatre ans de Directoire (1795-1799). Trois périodes de la vie parisienne de Paine sont distinguées. La première période (juin 1791-décembre 1793) s'étend de son investissement grandissant dans les débats français, alors que le roi Louis XVI a fui à Varennes, et de son installation à Paris en 1792 (il est élu député du Pas de Calais et cherche à échapper à la justice britannique) jusqu'à sa prise de position, des bancs de la Convention, contre la mort du roi et son emprisonnement à la prison du Luxembourg. La deuxième période (novembre 1794-août 1795) part de la sortie de prison de Paine et le montre participant activement aux débats portant sur la constitution de l'an III, débats au cours desquels il se fait remarquer par son opposition au rétablissement de la franchise censitaire. La troisième période est la plus « étrange et inattendue » (19); elle couvre la participation de Paine, en 1799, au coup d'État du 18 Brumaire et à l'installation du Consulat. Ce découpage donne une place particulière à l'année 1793, qui, pour Carine Lounissi, est un tournant dans la carrière comme dans la pensée politiques de Paine, une sorte de basculement.

Carine Lounissi établit une liste, qui se veut exhaustive et est en partie commentée, des 42 textes rédigés ou attribués par Paine au cours de cette période (21-25). La liste met en relief le fait que plusieurs des plus grands textes de Paine - Rights of Man (1791-1792), The Age of Reason (1794-1795) ou encore Agrarian Justice (1797) - furent rédigés au cours de cette période. C'est en quelque sorte l'œuvre de maturité de Paine, qui quitte la France alors qu'il a 65 ans. La liste comprend des titres qui ne figurent ni dans l'édition de Moncure Conway ni dans celle de Philip Foner, en particulier des articles publiés dans le Bien informé de Nicolas de Bonneville, un texte perdu comme «Observations on the Commerce between the United States of America and France " (1793) ou encore des textes attribués à Paine par erreur, en 1793 encore, volontairement ou non, comme une " Déclaration des droits de l'homme et du citoyen ». Elle ne comprend pas, en revanche, sans que Carine Lounissi ne s'en explique, la Lettre aux théophilanthropes, attribuée à Thomas Paine depuis ce qu'en a dit l'abbé Henri Grégoire dans son Histoire des sectes religieuses. Par ailleurs, de manière tout à fait intéressante, voire vertigineuse, la liste inclut aussi les textes du «Paine français », de ce Paine qui n'a pas existé, à savoir un auteur de textes en français. Seule la traduction française est en effet connue de 19 des textes attribués à Paine par Carine Lounissi ou d'autres historiens, par exemple ses «Réponses à quatre questions » publiées dans la Chronique du mois en 1792; ou encore son discours contre la mort du roi, lu à l'assemblée le 19 janvier 1793, texte qui fut peut-être traduit en anglais à partir de la version française, elle-même une traduction ; ou bien les six articles parus dans le Bien informé de Nicolas de Bonneville en 1797. À ces textes, il faut ajouter les textes en anglais perdus et retraduits par les historiens et biographes dont Carine Lounissi rend compte au fil des nombreuses notes qui concluent chaque chapitre de son livre. Pour établir cette liste, Carine Lounissi ne s'est pas contentée de se reposer sur les sources secondaires; elle a fouillé les archives nationales ainsi que d'" autres bibliothèques en France » (21). Des références précises aux archives et à ces bibliothèques auraient donné plus de force encore à l'établissement de cette liste et donc à l'entreprise de rectification menée par l'auteure.

4 Car, s'il ressort du livre qu'il reste encore des trous, des creux, des silences à combler, Carine Lounissi entend tout particulièrement mettre fin aux « conjectures fondées sur un vide documentaire», souvent le fait des biographes, mais pas uniquement. Dans ce 
but, elle réexamine un certain nombre de faits établis par les historiens, et ce, à la lecture des journaux de l'époque, qu'elle semble avoir littéralement épluchés, ainsi que des archives, surtout parlementaires, et des textes connus de Paine. Paine soutenait-il la monarchie française telle qu'elle avait été transformée après la fuite du roi en 1791 ? Rien ne le prouve, nous dit Carine Lounissi, pour qui l'implication de Paine dans la vie politique française ne commence vraiment qu'au cours de l'automne 1792, c'est-à-dire après son installation à Paris (98). Le désaccord entre Paine et Sieyès sur la compatibilité entre la monarchie et le système représentatif était-il de façade comme ont $\mathrm{pu}$ le dire plusieurs historiens de la révolution française? Non, conclut Carine Lounissi à la lumière des nouvelles archives; la critique de Sieyès à l'égard de la république telle que la concevait Paine n'était en rien arrangée (109). On a là une recherche minutieuse qui cherche à établir avec précision quelle fut la vie publique de Paine au cours de la période en question.

5 La contribution majeure de Carine Lounissi à la littérature existante est ici la proposition d'étudier les idées de Paine à la lumière du contexte français mais aussi de leur interaction avec les événements (6). Et cette méthodologie la conduit à revoir certaines des conclusions du livre issu de sa thèse, La pensée politique de Thomas en contexte. Théorie et pratique, publié par Champion en 2012, sans que l'on sache tout à fait lesquelles (4). Elle signale à plusieurs reprises que tel ou tel texte a fait l'objet d'études poussées, mais la plupart du temps dans un contexte autre que français. L'un des exemples les plus flagrants est peut-être Agrarian Justice, publié en anglais en avril 1797, texte souvent relevé pour ce que Paine y dit de l'aide sociale et du partage des biens communs, mais qui est, vu sous l'angle français, pointé pour avoir été une critique sévère de Babeuf et de la "Conjuration des Égaux " (la tentative de renversement du Directoire menée par Gracchus Babeuf et ses amis), et une apologie de la situation politique et économique de la France - cela alors que, souligne Carine Lounissi, Paine et Babeuf partageaient de nombreuses idées, et que la situation économique de la France n'était pas si bonne que cela (255). Carine Lounissi propose alors une analyse très intéressante, et originale, qui met en avant le besoin de Paine de penser la violence en république - besoin qui se fait sentir à plusieurs reprises : il s'agit là en effet d'une des nouvelles problématiques auxquelles Paine, comme ses contemporains, se trouve confronté. Que penser de la violence et comment la penser dans le contexte républicain postrévolutionnaire? Carine Lounissi va jusqu'à suggérer que Paine conclut sur la nécessité d'une certaine violence d'État: "[Paine's French period] obliged [him] to decide how violence should be handled by a republican governement, which could claim a potentially legitimate monopoly of violence against divergent opinions, which it said jeopardized its very existence » (20).

6 L'assertion selon laquelle Paine était girondin, question, nous rappelle Carine Lounissi, toujours débattue par les historiens, est, elle aussi, examinée à la loupe. L'auteure s'y attelle avec circonspection et de manière interrogative, établissant trois «moments girondins " de la vie de Paine à Paris. Car décrire Paine comme un girondin est problématique dans la mesure où il n'existait pas de groupe girondin homogène, unifié. L'auteure met en avant le réseau et les connexions établis par Paine avec Condorcet, Brissot, Bancal, Lanthenas (son traducteur), Bonneville (qui lui aussi traduisit des textes de Paine, et dont il fut de toute évidence le plus proche, et même ami, notamment sous le Directoire) et regarde de près les rapports que Paine eut avec ces hommes. Elle montre que si Paine a été défini comme un girondin par les historiens, il le fut d'abord par ses opposants. Ce n'est pourtant pas parce qu'il était girondin qu'il 
fut emprisonné à la fin de l'année 1793, mais parce qu'il était anglais. La proposition d'un deuxième "moment girondin" fut faite après sa libération, lorsque Paine retourna à la convention, en décembre 1794, et fut finalement réintégré comme "girondin ", non à la demande des girondins eux-mêmes mais de Thibaudeau qui plaide alors sa cause et le désigne comme «l'apôtre de la liberté » (222). Puis, il y a l'intégration de Paine dans le Cercle constitutionnel créé par Benjamin Constant and Mme de Staël en 1797. Ce troisième moment où Paine apparaît comme girondin est issu de la création, pour raisons politiques, de l'idée du girondin "martyr " mais aussi «modéré » (269).

7 Selon Carine Lounissi, trois idées centrales sont défendues par Paine. Le rejet du principe monarchique (ou anti-royalisme); la synonymie entre république et gouvernement représentatif (à l'instar de Condorcet, 106); le fait que le cadre constitutionnel est la sauvegarde la plus sûre du règne de la loi comme de la liberté politique et individuelle (p. 224). On connaît du Paine français son opposition à l'exécution du roi, ainsi que sa défense du suffrage universel masculin. Or, ce qui ressort du livre, c'est que la Révolution française, et en particulier les événements de 1793 mettent littéralement à l'épreuve les idées de Paine, voire en testent les limites (311). Les tensions partisanes, puis son emprisonnement, forcent l'auteur de Rights of Man à reconsidérer le lien entre souveraineté et participation, comme à lui faire prendre conscience du «fossé » qu'il y a entre constitutionalité et participation (200). Le Paine qui émerge du livre de Carine Lounissi est dès lors un Paine pragmatique (301), mais aussi un Paine propagandiste qui soutient indéfectiblement le Directoire (260). Carine Lounissi fait allusion à une forme de compromission lorsqu'elle analyse les prises de position de Paine dans la période thermidorienne et sous le Directoire : Paine alors reprend la «rhétorique de l'ordre » (264): «Paine's main purpose was to defend republicanism in a context in which it was threatened, even if it meant supporting a republican regime which was flawed in some respects ».

8 Le livre ainsi se conclut sur la perplexité dans laquelle plonge le Paine de ces années-là, un Paine qui défend comme Benjamin Constant des idées «libérales » c'est-à-dire qui considère que le gouvernement doit protéger les droits des individus contre tout pouvoir arbitraire tout en restreignant l'intervention des autorités publiques à son strict minimum («the governement should equally preserve the rights of individuals against any kind of arbitrariness and restrict the intervention of authorities to its minimum level »). Ce même Paine qui finalement se range aux côtés de ceux que nous pourrions appeler des «conservateurs", de ceux qui considèrent que la propriété privée est le socle du républicanisme, qui prônent la mise en place d'une « république de propriétaires» (312), de ceux qui vont jusqu'à préférer la mise en place d'une monarchie constitutionnelle (304). Carine Lounissi donne des raisons aux «retournements» de Paine: selon elle, l'allégeance au Directoire était un positionnement à l'égard des États-Unis, mais aussi à l'égard de la Grande-Bretagne, à savoir une façon de contrer les fédéralistes anti-français et pro-britanniques, et d'exprimer sa détestation de la monarchie britannique. Elle met aussi en avant la façon dont Paine se détourne d'une vieille Europe qui l'a déçu pour porter ses espoirs sur les États-Unis. En 1804, c'est l'exemple de 1776 qu'il met en avant alors qu'il s'adresse aux Britanniques, et non 1789 (313).

9 Le livre est consacré exclusivement à l'action politique de Paine. On peut regretter que la question religieuse n'y soit pas abordée, dans la mesure où la pensée de Paine, 
précisément, démontre assez bien l'imbrication du politique et du religieux. C'est dans le contexte français que Paine imagina et mit en œuvre la rédaction de son célèbre Âge of Reason, dont le premier tome se trouve justifié dès les premières lignes par les possibles excès de la politique religieuse de la république française ainsi que par la nécessité d'affirmer, et la croyance en un dieu unique et celle à l'égalité des êtres humain. Le silence vis-à-vis de la publication du Siècle de la raison - rien n'en est dit dans la presse - ou encore la tonalité très nettement anticléricale que la traduction de Bonneville donne à The Letter from Thomas Paine to Camille Jordan, of the Council of five hundred: Occasioned by his report of priests, public worship, and the bells, parue 1797, auraient pu fournir quelques éclairages. La critique religieuse de Paine ne semble pas avoir rencontré d'écho dans le contexte français alors que sa campagne en faveur du déisme apparaissait comme conforme aux intentions des Français qui cherchaient alors à donner une assise religieuse à la république - Bonneville publia L'Esprit des religions en 1791, Volney la Loi naturelle ou catéchisme du citoyen français en 1793, Condorcet Esquisse d'un tableau historique des progrès de l'esprit humain en 1794 - et que se mettaient en place des cultes déistes dans plusieurs villes de France. Le culte de la Raison puis celui de l'Être suprême furent mis en place en 1793 et 1794, avant que la théophilanthropie, dont Carine Lounissi ne dit rien, ne soit fondée à Paris en 1797, avec le soutien du directeur La Révellière-Lépeaux, et sans doute aussi celui de Paine. Est-ce à dire que Paine n'était pas lu et donc en décalage avec ses contemporains et compatriotes, ou bien, au contraire, qu'il était impliqué, selon un principe de distribution des rôles, dans une stratégie globale de dissémination des principes révolutionnaires mais aussi de mise en place d'une religion fondée sur la raison, en adéquation avec les principes bourgeois sur lesquels devait déboucher la révolution? Si l'on suit Carine Lounissi jusqu'au bout, peut-on alors voir dans la façon dont Paine s'est éloigné des affaires politiques une fois de retour aux États-Unis en 1802, pour se tourner vers des préoccupations surtout spirituelles, une lassitude vis-à-vis du politique, voire un dégoût, une sorte de crise existentielle qui l'aurait poussé vers la religion, cela d'autant plus qu'il doit alors faire face aux conflits partisans américains?

Carine Lounissi va parfois un peu trop loin, par exemple lorsqu'elle évoque un Paine « schizophrène » (312), ou un peu vite, lorsqu'elle affirme qu'après 1795, Paine « chose republicanism over democracy » (242). Un travail d'édition plus poussé de la part de Palgrave Macmillan aurait pu conduire à l'élimination de certaines maladresses, le lecteur buttant parfois sur les formules un peu confuses ou imprécises. Mais on a là un livre audacieux et dans l'ensemble tout à fait convaincant qui cherche à modeler une autre image de Thomas Paine, le démocrate radical, universaliste, cher à la gauche britannique et américaine, mais dont l'ambivalence - inhérente au libéralisme démocratique qu'il incarnait - ressort plus que jamais. L'argumentaire théorique de Thomas Paine, nous dit Carine Lounissi, se voit totalement bouleversé par son expérience française qui le pousse à renier certains de ces principes : « How legitimacy, sovereignty, participation and freedom could combine in a republican regime dramatically tossed Paine's theoretical boat to an extent unknown to him before he crossed the Atlantic again » (20.) Le Paine qui en ressort est un homme de l'expérience révolutionnaire, celle au cours de laquelle la philosophie politique se voit confrontée à la pratique de la politique et où de nouvelles questions - telle celle du rétablissement de l'ordre et de la légitimité de la violence - se posent. 
INDEX

Thèmes : Recensions

\section{AUTEURS}

NATHALIE CARON

Sorbonne Université 